\title{
Extended differential geometric guidance law for intercepting maneuvering targets
}

\author{
HUANG Jingshuai ${ }^{1}$, ZHANG Hongbo $^{1, *}$, TANG Guojian $^{1}$, and BAO Weimin ${ }^{2}$ \\ 1. College of Aerospace Science and Engineering, National University of Defense Technology, Changsha 410073, China; \\ 2. China Aerospace Science and Technology Corporation, Beijing 100048, China
}

\begin{abstract}
Without assumptions made on motion states of missile and target, an extended differential geometric guidance law is derived. Through introducing a line of sight rotation coordinate system, the derivation is simplified and has more explicit physical significances. The extended law is theoretically applicable to any engagement scenarios. Then, on basis of the extended law, a modified one is designed without the requirement of target acceleration and an approach is proposed to determining the applied direction of commanded missile acceleration. Qualitative analysis is carried out to study the capture performance and a criterion for capture is given. Simulation results indicate the two laws are effective and make up the deficiency that pure proportional navigation suitable for endoatmospheric interceptions cannot deal with high-speed maneuvering targets. Furthermore, the correctness of the criterion is validated.
\end{abstract}

Keywords: missile guidance, maneuvering target, extended differential geometric guidance law, capture performance, proportional navigation.

DOI: $10.21629 / J S E E .2018 .05 .15$

\section{Introduction}

Since the inception of proportional navigation $(\mathrm{PN})$ in the 1940s [1], a considerable amount of attention has been paid to PN in missile guidance literature such that PN has been developed into many different forms, such as pure PN (PPN), true PN (TPN), ideal PN (IPN), and augmented PN (APN) [2-5]. Due to inherent simplicity, ease of realization, and effectiveness, PN has been extensivelyapplied in engineering practices of missile guidance community. To realize specific intercept missions, in-depth studies on PN were implemented in [6-8]. However, the performance of $\mathrm{PN}$ drastically degrades as targets become smarter and stealthier. This is mainly because that PN has no enough capability to control line-of-sight (LOS) rotation induced by target maneuver, which brings about the

Manuscript received January 18, 2017.

*Corresponding author. divergence of missile overload. In order to capture an agile target, APN emerged, but the price paid was the information of target acceleration [5]. Moreover, scholars have been trying to investigate advanced guidance laws based on modern control theories and other methodologies that are expected to be superior to the classical PN laws.

Classical differential geometric (DG) theory is the use of calculus to quantify the change trend of space curve that is formulated as curvature and torsion parameters [9]. The missile guidance, in fact, is a planning problem of the trajectory curve, which indicates that the trajectory is determined provided that the curvature and torsion parameters are given. Therefore, the DG theory provides a different insight into missile guidance and corresponding plentiful and substantial achievements have been made. Additionally, it is gradually applied to path-following and near-distance rendezvous guidance for unmanned aerial vehicle and spacecraft, respectively, which manifests great advantages over traditional methods $[10,11]$. Under the assumptions that both missile and target moved at a constant velocity and the ratio of target was less than 1 to missile, Chiou et al. [12] initially studied the three-dimensional relative motion of missile and target based on the DG theory and put forward a three-dimensional differential geometric guidance law (DGGL) composed of curvature and torsion commands. Then, Kuo et al. [13,14] explored the planar form of DGGL. Li et al. [15-21] transformed DGGL into the corresponding form in time domain and were dedicated to combining DGGL with missile control system. $\mathrm{Li}$ et al. [22-24] proposed a generalized constructing approach to DGGL based on the relative motion equations described in a defined LOS rotation coordinate system, and found that the DGGL in [12] was a special case of the DG form of APN. Furthermore, the geometric and algebraic solutions were given to the applied direction of curvature command, which eliminated the complex torsion calculation and enhanced the robustness of DGGL. By intro- 
ducing sliding-mode control theory and Lie-group method into the DG model of missile-target relative motion, Ye et al. [25] designed a nonlinear DGGL which obtained better performance than the DGGL in [12] for agile targets. The main contribution of DGGL is that it provides a new application direction of commanded acceleration. Moreover, a designable guidance acceleration variable included in the generalized DGGL [22] can be made use of to create DGGLs whose efficiencies of controlling LOS rotation are enhanced. Aimed at the imperfection that traditional DGGLs could not guarantee the finite-time convergence to zero of LOS rate (LOSR), Ma et al. [26,27] used finite-time stability theory to design a finite-time-convergence DGGL based on the generalized DGGL [24] and target acceleration was estimated. In addition, Ariff et al. [28] proposed a DGGL based on the involute of target trajectory. White et al. [29] presented another DGGL that offered a suite of possible missile trajectories to intercept target. For simplicity, the most studies about DGGL are based on the two assumptions made in [12], which largely limits the application range of DGGL. While [22] provided the generalized DGGL and the improved form was given in [23], the kinematic and dynamic analyses were not addressed of the DG guidance problem.

This paper differs from prior work in three main aspects. First, by virtue of the LOS rotation coordinate system, the DG guidance problem is explored under the condition that no assumptions are made on motion states of missile and target and an extended DGGL (EDGGL) is obtained applicable to engagement situations where target maneuvers arbitrarily and the velocity of missile is not necessarily greater than that of target. Second, instead of torsion command, a novel and simple approach is presented to determine the applied direction of commanded missile acceleration when target acceleration is difficult to acquire, and then a modified EDGGL (MEDGGL) is achieved. Third, a capture criterion is given via analyzing total cases.

The rest of this paper is organized as follows: Section 2 presents the DG model of interception. In Section 3, the derivation of EDGGL is performed. Further efforts are made to obtain MEDGGL in Section 4. Section 5 qualitatively conducts the analysis of capture performance. Simulations are carried out in Section 6. In the last Section, conclusions are drawn.

\section{DG modeling of interception}

Before modeling, a brief introduction is given into the classical DG theory. With regard to an arbitrary smooth space curve, we can establish the Frenet frame at any point. As shown in Fig. $1, \boldsymbol{t}, \boldsymbol{n}$, and $\boldsymbol{b}$ represent the tangential, normal and binormal unit vectors, respectively, and constitute the Frenet frame [9]. Their definitions are given by

$$
\left\{\begin{array}{l}
\boldsymbol{t}=\boldsymbol{r}^{\prime}=\frac{\mathrm{d} \boldsymbol{r}}{\mathrm{d} s} \\
\boldsymbol{n}=\boldsymbol{t}^{\prime} /\left|\boldsymbol{t}^{\prime}\right| \\
\boldsymbol{b}=\boldsymbol{t} \times \boldsymbol{n}
\end{array}\right.
$$

where $\mathrm{d} s$ denotes the differential with respect to the arc length of curve. According to (1), we can obtain the essential relations written as the following Frenet formula [9]:

$$
\left\{\begin{array}{l}
\frac{\mathrm{d} t}{\mathrm{~d} s}=\kappa \boldsymbol{n} \\
\frac{\mathrm{d} n}{\mathrm{~d} s}=-\kappa \boldsymbol{t}+\tau \boldsymbol{b} \\
\frac{\mathrm{d} b}{\mathrm{~d} s}=-\tau \boldsymbol{n}
\end{array}\right.
$$

where $\kappa$ and $\tau$ are the curvature and torsion of curve, respectively. Assuming the velocity of point mass is $v$, we have the following differential relation:

$$
\mathrm{d} s=v \mathrm{~d} t
$$

where $\mathrm{d} t$ is the differential with respect to time.

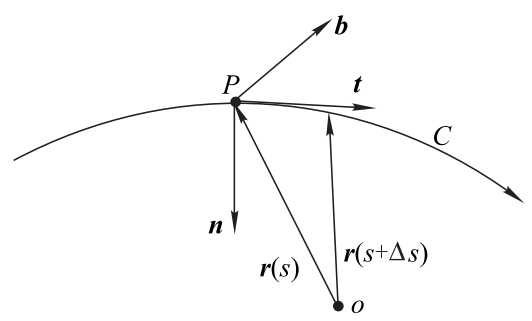

Fig. 1 Frenet frame

The three-dimensional engagement geometry is depicted in Fig. 2.

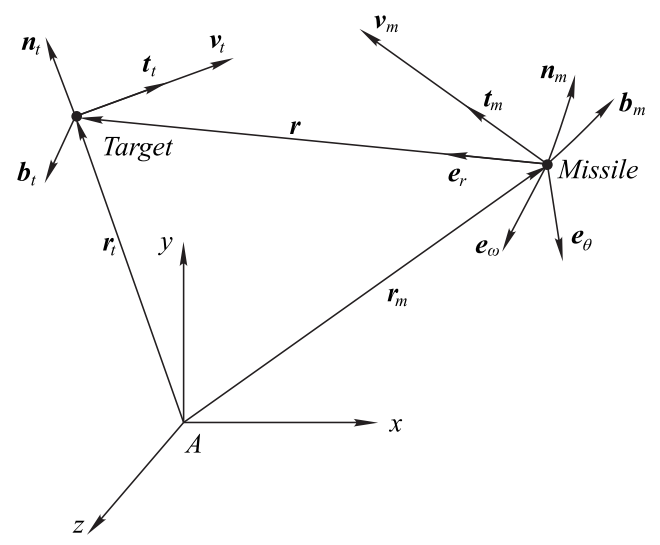

Fig. 2 Missile-target engagement geometry

Considering the relative velocity between missile and target is rapid and the engagement time is short in the terminal phase of interception, the rotation and the curvature of Earth are ignored and the ground coordinate system Axyz is regarded as inertial. Here, we just assume that 
the missile and the target are point masses with no restrictions on their motion states. In three-dimensional endoatmospheric engagements, the trajectories of missile and target can be treated as smooth space curves approximately.

In Fig. 2, $\left(\boldsymbol{t}_{m}, \boldsymbol{n}_{m}, \boldsymbol{b}_{m}\right)$ and $\left(\boldsymbol{t}_{t}, \boldsymbol{n}_{t}, \boldsymbol{b}_{t}\right)$ represent the Frenet frames of missile and target, respectively. Therefore, the Frenet formula (2) is satisfied. Because the rotation of LOS is absolutely dependent on the components normal to the LOS of $\boldsymbol{v}_{m}$ and $\boldsymbol{v}_{t}$, the unit vector of rotational angular velocity of $\operatorname{LOS} e_{\omega}$ is also normal to the LOS [12]. That is, we have

$$
\boldsymbol{e}_{\omega} \cdot \boldsymbol{e}_{r}=0
$$

Also, the unit vector $\boldsymbol{e}_{\theta}$ is defined as

$$
e_{\theta}=e_{\omega} \times e_{r}
$$

Then, $\left(\boldsymbol{e}_{r}, \boldsymbol{e}_{\theta}, \boldsymbol{e}_{\omega}\right)$ constitutes the LOS rotation coordinate system whose origin is located in the mass center of missile. Based on its definition, the relations analogous to (2) are derived [22]:

$$
\left\{\begin{array}{l}
\frac{\mathrm{d} \boldsymbol{e}_{r}}{\mathrm{~d} t}=\omega_{t} \boldsymbol{e}_{\theta} \\
\frac{\mathrm{d} \boldsymbol{e}_{\theta}}{\mathrm{d} t}=-\omega_{t} \boldsymbol{e}_{r}+\Omega_{t} \boldsymbol{e}_{\omega} \\
\frac{\mathrm{d} \boldsymbol{e}_{\omega}}{\mathrm{d} t}=-\Omega_{t} \boldsymbol{e}_{\theta}
\end{array}\right.
$$

where $\omega_{t}$ and $\Omega_{t}$ are the magnitudes of angular velocity of $\boldsymbol{e}_{r}$ and $\boldsymbol{e}_{\omega}$, respectively. In addition,

$$
\omega_{t} \geqslant 0, \quad\left|\Omega_{t}\right| \geqslant 0
$$

The relative motion equations described in $\left(\boldsymbol{e}_{r}, \boldsymbol{e}_{\theta}, \boldsymbol{e}_{\omega}\right)$ [22] can be expressed as

$$
\left\{\begin{array}{l}
\ddot{r}-r \omega_{t}^{2}=a_{t r}-a_{m r} \\
2 \dot{r} \omega_{t}+r \dot{\omega}_{t}=a_{t \theta}-a_{m \theta} \\
r \omega_{t} \Omega_{t}=a_{t \omega}-a_{m \omega}
\end{array}\right.
$$

where $a$ represents the acceleration; the subscripts ' $t$ ' and ' $m$ ' denote the scalar quantities belonging to target and missile, respectively; the subscripts ' $r$ ', ' $\theta$ ', and ' $\omega$ ' denote the scalar components along $\boldsymbol{e}_{r}, \boldsymbol{e}_{\theta}$, and $\boldsymbol{e}_{\omega}$, respectively.

\section{Derivation of extended DGGL}

From Fig. 2, we have

$$
\boldsymbol{r}=\boldsymbol{r}_{t}-\boldsymbol{r}_{m}=\boldsymbol{r} \boldsymbol{e}_{r}
$$

Taking the derivative of (9) with respect to the arc length of missile trajectory $s_{m}$ gives

$$
\frac{v_{t}}{v_{m}} \boldsymbol{t}_{t}-\boldsymbol{t}_{m}=r^{\prime} \boldsymbol{e}_{r}+r \omega \boldsymbol{e}_{\theta}
$$

where $\omega=\omega_{t} / v_{m}$. Then, the scalar components of (10) along $\boldsymbol{e}_{r}$ and $\boldsymbol{e}_{\theta}$ are

$$
\begin{aligned}
r^{\prime} & =\left(\frac{v_{t}}{v_{m}} \boldsymbol{t}_{t}-\boldsymbol{t}_{m}\right) \cdot \boldsymbol{e}_{r} \\
r \omega & =\left(\frac{v_{t}}{v_{m}} \boldsymbol{t}_{t}-\boldsymbol{t}_{m}\right) \cdot \boldsymbol{e}_{\theta} .
\end{aligned}
$$

Taking the derivative of (10) with respect to $s_{m}$, we have the basic dynamics equation

$$
\begin{gathered}
\left(r^{\prime \prime}-r \omega^{2}\right) \boldsymbol{e}_{r}+\left(2 r^{\prime} \omega+r \omega^{\prime}\right) \boldsymbol{e}_{\theta}+r \omega \Omega \boldsymbol{e}_{\omega}= \\
\left(\frac{v_{t}}{v_{m}}\right)^{\prime} \boldsymbol{t}_{t}+\left(\frac{v_{t}}{v_{m}}\right)^{2} \kappa_{t} \boldsymbol{n}_{t}-\kappa_{m} \boldsymbol{n}_{m}
\end{gathered}
$$

where $\Omega=\Omega_{t} / v_{m}$, and $\kappa_{m}$ and $\kappa_{t}$ are the curvatures of missile and target trajectories, respectively.

To achieve little miss distance, it is expected that LOSR is zero, which implies the velocity component normal to LOS of missile is equal to that of target. It is assumed that there exists a certain moment when the instantaneous LOSR is null. Then, the corresponding fictitious missile velocity vector is denoted by $\boldsymbol{v}_{m p}$. From (10), we consequently have

$$
\frac{v_{t}}{v_{m}} \boldsymbol{t}_{t}-\boldsymbol{t}_{m p}=r_{m p}^{\prime} \boldsymbol{e}_{r}
$$

where $\boldsymbol{v}_{m p}=\boldsymbol{v}_{m}$ holds in that the commanded missile acceleration is normal to the missile velocity in DGGL. Then, due to (14), we have

$$
\left(\frac{v_{t}}{v_{m}} \boldsymbol{t}_{t}-\boldsymbol{t}_{m p}\right) \cdot \boldsymbol{e}_{\theta}=0
$$

Before proceeding with the derivation, it is necessary to study the existence condition for $\boldsymbol{v}_{m p}$. Fig. 3 is used to describe the relations of spatial orientations among $\boldsymbol{v}_{m}, \boldsymbol{v}_{t}$, and $\boldsymbol{v}_{m p}$.

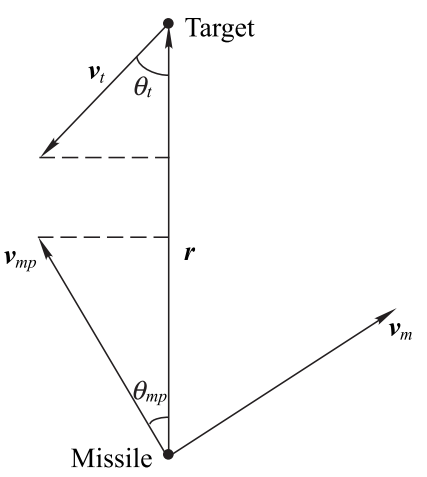

Fig. 3 Spatial orientations of velocity vectors

According to Fig. 3, we have

$$
\boldsymbol{v}_{m p}-\left(\boldsymbol{v}_{m p} \cdot \boldsymbol{e}_{r}\right) \boldsymbol{e}_{r}=\boldsymbol{v}_{t}-\left(\boldsymbol{v}_{t} \cdot \boldsymbol{e}_{r}\right) \boldsymbol{e}_{r} .
$$

Then, the following relation is obtained:

$$
v_{m}=v_{m p} \geqslant v_{m p} \sin \theta_{m p}=v_{t} \sin \theta_{t} .
$$


Thus, if $v_{m} \geqslant v_{t}$ holds, then $\boldsymbol{v}_{m p}$ definitely exists.

Taking the derivative of (15) with respect to $s_{m}$ and applying the second equation of (6), we have

$$
\begin{gathered}
{\left[\left(\frac{v_{t}}{v_{m}}\right)^{\prime} \boldsymbol{t}_{t}+\left(\frac{v_{t}}{v_{m}}\right)^{2} \kappa_{t} \boldsymbol{n}_{t}-\kappa_{m p} \boldsymbol{n}_{m p}\right] \cdot \boldsymbol{e}_{\theta}+} \\
\left(\frac{v_{t}}{v_{m}} \boldsymbol{t}_{t}-\boldsymbol{t}_{m p}\right) \cdot\left(-\omega \boldsymbol{e}_{r}+\Omega \boldsymbol{e}_{\omega}\right)=0
\end{gathered}
$$

By substituting (14) into (18), (18) is converted into

$$
\kappa_{m p}=\frac{\left[\left(\frac{v_{t}}{v_{m}}\right)^{\prime} \boldsymbol{t}_{t}+\left(\frac{v_{t}}{v_{m}}\right)^{2} \kappa_{t} \boldsymbol{n}_{t}\right] \cdot \boldsymbol{e}_{\theta}}{\boldsymbol{n}_{m p} \cdot \boldsymbol{e}_{\theta}}-\frac{r_{m p}^{\prime} \omega}{\boldsymbol{n}_{m p} \cdot \boldsymbol{e}_{\theta}}
$$

which is referred to as the curvature command corresponding to $\boldsymbol{v}_{m p}$. However, the actual missile velocity vector is $\boldsymbol{v}_{m}$. By substituting $r^{\prime}$ and $\boldsymbol{n}_{m}$ for $r_{m p}^{\prime}$ and $\boldsymbol{n}_{m p}$, respectively, and adding a proportional constant $N$ into the second term of (19), the curvature command corresponding to $\boldsymbol{v}_{m}$ is constructed:

$$
\kappa_{m}=\frac{\left[\left(\frac{v_{t}}{v_{m}}\right)^{\prime} \boldsymbol{t}_{t}+\left(\frac{v_{t}}{v_{m}}\right)^{2} \kappa_{t} \boldsymbol{n}_{t}\right] \cdot \boldsymbol{e}_{\theta}-N r^{\prime} \omega}{\boldsymbol{n}_{m} \cdot \boldsymbol{e}_{\theta}} .
$$

Due to the application direction of curvature in line with $\boldsymbol{n}_{m}$, the missile acceleration vector $\boldsymbol{a}_{m}$ is written as

$$
\boldsymbol{a}_{m}=a_{m} \boldsymbol{n}_{m}=v_{m}^{2} \kappa_{m} \boldsymbol{n}_{m} .
$$

Substituting (20) into (13), we have

$$
2 r^{\prime} \omega+r \omega^{\prime}=N r^{\prime} \omega
$$

By solving (22), the analytical solution to LOSR is achieved:

$$
\omega=\omega_{0}\left(r / r_{0}\right)^{N-2} .
$$

From (23), if $N>2$, LOSR will gradually decreases to zero as the missile is more and more closer to the target, which signifies the formation of a collision course. Therefore, $N>2$ is indispensable to interception. It can be seen that (23) holds regardless of the sufficient existence condition for $\boldsymbol{v}_{m p}$ as long as the missile curvature command satisfies (20).

In (20), it is possible that the curvature command becomes singular during engagement, i.e., $\boldsymbol{n}_{m} \cdot \boldsymbol{e}_{\theta}=0$. Therefore, to ensure the curvature command is well defined, we can [12] assume

$$
\boldsymbol{n}_{m} \cdot \boldsymbol{e}_{\theta}=\lambda
$$

where $0<\lambda \leqslant 1$. Taking the derivative of (24) with respect to $s_{m}$ and applying the Frenet formula, we have the torsion command expressed as

$$
\tau_{m}=\left|\kappa_{m}\right| \frac{\boldsymbol{t}_{m} \cdot \boldsymbol{e}_{\theta}}{\boldsymbol{b}_{m} \cdot \boldsymbol{e}_{\theta}}+\omega \frac{\boldsymbol{n}_{m} \cdot \boldsymbol{e}_{r}}{\boldsymbol{b}_{m} \cdot \boldsymbol{e}_{\theta}}-\Omega \frac{\boldsymbol{n}_{m} \cdot \boldsymbol{e}_{\omega}}{\boldsymbol{b}_{m} \cdot \boldsymbol{e}_{\theta}}
$$

where $\left|\kappa_{m}\right|$ is adopted since $\kappa$ is nonnegative in the Frenet formula and $\kappa_{m}$ calculated from (20) may be negative. The torsion $\tau_{m}$ in (25) is used to adjust the direction of $\boldsymbol{n}_{m}$ for the purpose of keeping $\boldsymbol{n}_{m} \cdot \boldsymbol{e}_{\theta}$ constant. According to the third equation of (8), we obtain

$$
\Omega=\frac{\left(\boldsymbol{a}_{t}-\boldsymbol{a}_{m}\right) \cdot \boldsymbol{e}_{\omega}}{r \omega_{t} v_{m}} .
$$

Through the above derivation, the curvature and torsion commands are achieved. Then, (20) and (25) constitute the EDGGL. Because there are no limitations on motion states of missile and target, EDGGL can be suitable for any engagement scenarios irrespective of its practical capture capability and available missile overload, which significantly extends the application range of DGGL. For simplicity in simulation, by (3) EDGGL is transformed into the form of time domain:

$$
\begin{gathered}
\kappa_{m}=\frac{1}{v_{m}^{2}} \frac{\boldsymbol{a}_{t} \cdot \boldsymbol{e}_{\theta}-N \dot{r} \omega_{t}}{\boldsymbol{n}_{m} \cdot \boldsymbol{e}_{\theta}} \\
\tau_{m}=\left|\kappa_{m}\right| \frac{\boldsymbol{t}_{m} \cdot \boldsymbol{e}_{\theta}}{\boldsymbol{b}_{m} \cdot \boldsymbol{e}_{\theta}}+\frac{\omega_{t}}{v_{m}} \frac{\boldsymbol{n}_{m} \cdot \boldsymbol{e}_{r}}{\boldsymbol{b}_{m} \cdot \boldsymbol{e}_{\theta}}-\frac{\Omega_{t}}{v_{m}} \frac{\boldsymbol{n}_{m} \cdot \boldsymbol{e}_{\omega}}{\boldsymbol{b}_{m} \cdot \boldsymbol{e}_{\theta}} .
\end{gathered}
$$

Meanwhile, PPN, TPN and augmented TPN (ATPN) $[22,23]$ are defined as

$$
\left\{\begin{array}{l}
\boldsymbol{a}_{m \mathrm{PPN}}=N v_{m} \boldsymbol{\omega}_{t} \times \boldsymbol{t}_{m} \\
\boldsymbol{a}_{m \mathrm{TPN}}=a_{m \mathrm{TPN}} \boldsymbol{e}_{\theta}=-N \dot{r} \omega_{t} \boldsymbol{e}_{\theta} \\
\boldsymbol{a}_{m \mathrm{ATPN}}=a_{m \mathrm{ATPN}} \boldsymbol{e}_{\theta}=\left(a_{t \theta}-N \dot{r} \omega_{t}\right) \boldsymbol{e}_{\theta}
\end{array} .\right.
$$

By substituting ATPN in (29) into the second equation of (8), ATPN has the same relation as EDGGL, that is,

$$
\omega_{t}=\omega_{t 0}\left(r / r_{0}\right)^{N-2} .
$$

Combining the ATPN expression and (27) yields

$$
a_{m}=\frac{a_{m \mathrm{ATPN}}}{\boldsymbol{n}_{m} \cdot \boldsymbol{e}_{\theta}}
$$

However, in TPN and ATPN, the commanded missile acceleration is applied normal to LOS, while, in EDGGL and PPN, the acceleration normal to missile velocity. As a whole, in consideration of differences in actuator, the guidance laws normal to LOS and missile velocity are appropriate for exoatmospheric and endoatmospheric intercepts, respectively.

\section{Modified EDGGL}

From (27) and (28), it can be seen that the commands generated by EDGGL need target acceleration information. However, there may be no way to obtain the information or the obtained is inaccurate in most cases. Moreover, the information with errors substituted into (27) and (28), 
particularly (28) whose form is complicated, may trigger the oscillation of guidance command, which is quite detrimental to the actuator. Hence, we are motivated to modify EDGGL referring to TPN in which the acceleration compensation term is directly removed from ATPN.

On basis of the above, without target acceleration available the curvature command (27) is modified into the following form:

$$
\kappa_{m}=\frac{1}{v_{m}^{2}} \frac{-N \dot{r} \omega_{t}}{\boldsymbol{n}_{m} \cdot \boldsymbol{e}_{\theta}} .
$$

Meanwhile, no acceleration information means the torsion command (28) cannot be calculated, which forces us to look for another method to determine $\boldsymbol{n}_{m}$. In fact, we should realize that in DGGLs the following equations have to be met:

$$
\left\{\begin{array}{l}
\boldsymbol{n}_{m} \cdot \boldsymbol{t}_{m}=0 \\
\boldsymbol{n}_{m} \cdot \boldsymbol{e}_{\theta}=\lambda . \\
\left|\boldsymbol{n}_{m}\right|=1
\end{array} .\right.
$$

By setting $\boldsymbol{n}_{m}=(x, y, z), \boldsymbol{t}_{m}=\left(a_{1}, a_{2}, a_{3}\right)$, and $\boldsymbol{e}_{\theta}=$ $\left(b_{1}, b_{2}, b_{3}\right)$, the existence condition for $\boldsymbol{n}_{m}$ is achieved:

$$
A^{2}+B^{2}+C^{2} \geqslant \lambda^{2}
$$

where

$$
A=\left|\begin{array}{ll}
a_{1} & a_{2} \\
b_{1} & b_{2}
\end{array}\right|, \quad B=\left|\begin{array}{ll}
a_{1} & a_{3} \\
b_{1} & b_{3}
\end{array}\right|, \quad C=\left|\begin{array}{ll}
a_{2} & a_{3} \\
b_{2} & b_{3}
\end{array}\right| .
$$

During interceptions, (34) may be no longer satisfied since $A, B$ and $C$ change in real time. Thus, we should properly lower $\lambda$. Here, we can select it as

$$
\lambda=\sqrt{A^{2}+B^{2}+C^{2}}-\delta
$$

where $\delta>0$ is sufficiently small.

Solving (33), we generally obtain two solutions denoted by $\boldsymbol{n}_{m 1}$ and $\boldsymbol{n}_{m 2}$. In order to guarantee the command continuity, the solution is chosen which satisfies the following condition:

$$
\boldsymbol{n}_{m}=\left\{\boldsymbol{n}_{m i} \mid \max \left[\cos <\boldsymbol{n}_{m i}, \boldsymbol{n}_{m}^{\prime}>\right] ; i=1,2\right\}
$$

where $\boldsymbol{n}_{m}^{\prime}$ is the previous moment of $\boldsymbol{n}_{m}$. Thus, MEDGGL is obtained without complex calculations for torsion. Due to no acceleration compensation in MEDGGL, (23) holds only when $a_{t \theta}=0$. Similarly, according to (32) and the TPN in (29), we have

$$
a_{m \mathrm{MEDGGL}}=\frac{a_{m \mathrm{TPN}}}{\boldsymbol{n}_{m} \cdot \boldsymbol{e}_{\theta}} .
$$

\section{Analysis of capture performance}

According to the preceding section, for MEDGGL when $a_{t \theta}=0$ or EDGGL, (23) is satisfied. Hence, there are two conclusions: i) $\omega>0$ holds before $r=0$; ii) $r$ and $\omega$ have the same variability trend, that is, $\omega^{\prime}<0$ if $r^{\prime}<0$. Therefore, if $r^{\prime}<0$ is guaranteed, then before collision the differential of $r \omega$ with respect to $s_{m}$ yields

$$
\frac{\mathrm{d}}{\mathrm{d} s_{m}}(r \omega)=r^{\prime} \omega+r \omega^{\prime}<0 .
$$

From (38), $r \omega$ will gradually decrease during engagement.

Due to $r \geqslant 0$, the values of $r$ and $r^{\prime}$ can be divided into the following six possibilities: i) $r>0, r^{\prime}<0$; ii) $r>0$, $r^{\prime}=0$; iii) $r>0, r^{\prime}>0$; iv) $r=0, r^{\prime}<0$; v) $r=0$, $r^{\prime}=0$; vi) $r=0, r^{\prime}>0$. In addition, in order to intercept targets in the terminal course, the initial $r^{\prime}$ is usually set as negative.

Case 1 Due to (22), $r^{\prime}<0$ can deduce $\omega^{\prime}<0$. It is indicated that the missile is flying to the target and a collision course is forming.

Case $2 \omega>0$ and $\omega^{\prime}=0$ can be obtained from (23) and (22), respectively. Those mean missing targets are quite possible to occur, which is an indication of interception failure.

Case 3 Because $r$ is continuous, this situation is bound to appear after Case 2.

Case 4 It is expected that the sign of $r^{\prime}$ is unchanged until $r$ is zero.

Case 5 Setting $r=0$ and $r^{\prime}=0$ in (10), we have $\boldsymbol{v}_{t}=\boldsymbol{v}_{m}$. In practical interceptions, the relation is almost impossible.

Case 6 Since $r$ is continuous and its minimum value is zero, $r^{\prime}>0$ at $r=0$ is contradictory.

Based on the above analysis, the sufficient condition to ensure capture is keeping the sign of $r^{\prime}$ unchanged or suppressing the occurrence of Case 2 during engagement. If this requirement is fulfilled, Case 1 is eventually converted into Case 4.

Now, it is assumed that an interception finally fails. Accordingly, Case 2 is bound to emerge in some moment. The corresponding velocity vectors of missile and target are denoted by $\boldsymbol{v}_{m e}$ and $\boldsymbol{v}_{t e}$, respectively. Substituting Case 2 into (10), we have

$$
(r \omega)_{m e} \boldsymbol{e}_{\theta}=\frac{v_{t e}}{v_{m}} \boldsymbol{t}_{t e}-\boldsymbol{t}_{m e} .
$$

Due to $(r \omega)_{m e}>0, \boldsymbol{e}_{\theta}$ is in the same direction as $\left(v_{t e} / v_{m e}\right) \boldsymbol{t}_{t e}-\boldsymbol{t}_{m e}$. Then,

$$
\left[(r \omega)_{m e}\right]_{\min }=\left|\frac{v_{t e}}{v_{m}} \boldsymbol{t}_{t e}-\boldsymbol{t}_{m e}\right|_{\min }=\left|\frac{v_{t e}}{v_{m}}-1\right| .
$$

In (40), the conditions of minimum values are divided into two cases: i) when $v_{t e} \geqslant v_{m}, \boldsymbol{t}_{m e}=\boldsymbol{t}_{t e}=\boldsymbol{e}_{\theta}$; ii) when 
$v_{t e} \leqslant v_{m}, \boldsymbol{t}_{m e}=\boldsymbol{t}_{t e}=-\boldsymbol{e}_{\theta}$. If the initial $r \omega$ satisfies the following inequality:

$$
r_{0} \omega_{0}<\left[(r \omega)_{m e}\right]_{\min }=\left|\frac{v_{t e}}{v_{m}}-1\right|,
$$

then, because of (38), $r \omega$ will monotonously decrease to zero and always be less than $\left[(r \omega)_{m e}\right]_{\min }$. This implies that miss does not occur and capture happens when $r=0$. In addition, it can be seen that in practical interceptions the probability is nearly zero that the minimum conditions are satisfied. Therefore, the requirement of (41) is conservative. The above analysis for capture only imposes a restriction on $r_{0} \omega_{0}$ and the capture capability in practical interceptions is subjected to many other factors.

\section{Simulations}

In this section, simulations are divided into two parts. One is to demonstrate the interception performance of EDGGL and MEDGGL against maneuvering targets, and the other is for validating the reasonability of (41).

\subsection{Performance demonstration of DGGL}

By numerical simulations, EDGGL and MEDGGL are tested and verified in three-dimensional pursuing and headon intercepting scenarios. Meanwhile, they are compared with TPN, ATPN and PPN. The command lag of the missile is taken into account and modeled as a first-order differential equation in the form of

$$
\dot{a}_{m a}=-\frac{1}{\alpha} a_{m a}+\frac{1}{\alpha} a_{m c}
$$

where $a_{m a}$ and $a_{m c}$ are the actual and commanded accelerations, respectively, and $\alpha$ is the time constant. To evaluate the overload consumption of the guidance law, the cumulative velocity increment $\Delta v$ of missile during interception is defined as

$$
\Delta v=\int_{0}^{t_{f}}|\boldsymbol{a}| \mathrm{d} t .
$$

where $t_{f}$ is the action time of the guidance law, and $|\boldsymbol{a}|$ is the magnitude of missile acceleration. In addition, $N=3$, $\delta=0.05$, and $g_{0}=9.80665 \mathrm{~m} / \mathrm{s}^{2}$ are adopted. The simulation step is $0.001 \mathrm{~s}$ when $r>5 \mathrm{~km}$, and $0.0001 \mathrm{~s}$ when $r \leqslant 5 \mathrm{~km}$. The simulation is terminated when $\dot{r}>0$.

Scenario A The missile with a velocity advantage pursuing target. The initial states of missile and target in Axyz and two types of target maneuver are listed in Table 1.

Table 1 Simulation settings in Scenario A

\begin{tabular}{ccc}
\hline Setting & Missile & Target \\
\hline Initial position/km & $(1,20,1.5)$ & $(4,30,1.5)$ \\
Initial speed/(km/s) & 1 & 0.4 \\
Initial pointing of $\boldsymbol{t}$ & $(0.6395,0.7543,0.1486)$ & $(1,0,0)$ \\
Initial pointing of $\boldsymbol{n}$ & $(0.4726,-0.2333,-0.8498)$ & - \\
Maneuvering manner & - & $\mathrm{T}_{1}:\left(0,2 g_{0}, 3 g_{0}\right) ; \mathrm{T}_{2}:\left(0,2 g_{0}, 4 g_{0} \cos t\right)$ \\
\hline
\end{tabular}

From Table 1, the initial $\boldsymbol{n}_{m} \cdot \boldsymbol{e}_{\theta}$ is 0.8026 , and the initial $r \omega$ is

$$
r_{0} \omega_{0}=0.1491<\left|\frac{v_{t 0}}{v_{m}}-1\right|=0.6 .
$$

Obviously, (41) is satisfied. In (44), it is assumed that $v_{t}$ changes a little due to the short engagement time, and then the initial target velocity $v_{t 0}$ is approximately substituted for $v_{t e}$.

In the following simulations, the maximum overload of the missile is set as $8 g_{0}$, the seeker blind zone is defined as $100 \mathrm{~m}$, and the target successively adopts the maneuvering manners of $\mathrm{T}_{1}$ and $\mathrm{T}_{2}$. EDGGL and MEDGGL are used to guide the missile and, meanwhile, compared with TPN, ATPN and PPN. The simulation results are presented in Figs. 4-9 and Table 2.

According to Table 2 and Fig. 6 and Fig. 7, the overload consumptions of EDGGL and MEDGGL are more than those of ATPN and TPN, respectively, which are attributed to (31) and (37). In addition, it can be seen from $\mathrm{T}_{1}$ in Table 2 that, because of containing the compensation term of target maneuver, EDGGL has an obvious advantage over MEDGGL and, likewise, ATPN does over TPN. $\mathrm{T}_{2}$ in Table 2, however, indicates that the compensation term is inclined to increase the overload consumption.

Table 2 Comparisons of simulation results in Scenario A

\begin{tabular}{cccccc}
\hline \multirow{2}{*}{$\begin{array}{c}\text { Guidance } \\
\text { law }\end{array}$} & \multicolumn{2}{c}{$\mathrm{T}_{1}$} & & \multicolumn{2}{c}{$\mathrm{T}_{2}$} \\
\cline { 2 - 3 } \cline { 5 - 6 } & Miss & $\Delta v /(\mathrm{m} / \mathrm{s})$ & & $\begin{array}{c}\text { Miss } \\
\text { distance } / \mathrm{m}\end{array}$ & $\Delta v /(\mathrm{m} / \mathrm{s})$ \\
\hline EDGGL & 0.8937 & 453.6090 & & 0.5722 & 516.6550 \\
MEDGGL & 2.0458 & 678.6976 & & 1.1225 & 512.9672 \\
ATPN & 0.6214 & 382.1784 & & 0.6088 & 447.2789 \\
TPN & 1.8697 & 613.4991 & & 3.2835 & 397.7052 \\
PPN & 1.3584 & 571.5543 & & 0.9350 & 398.7429 \\
\hline
\end{tabular}

This is because, as shown in Fig. 7, that the sinusoidal maneuvering of the target leads to the higheramplitude oscillations of compensated guidance acceleration in EDGGL and ATPN than in MEDGGL and TPN, respectively. From Fig. 8 and Fig. 9, it is known that EDGGL and ATPN have the ability to reduce LOSR close to zero and the correctness of (23) and (30) is consequently 
proved. Since engagement scenarios analogous to Scenario A usually happen inside the atmosphere, we can conclude that PPN is an eclectic option on basis of comprehensively taking into account miss distance, overload consumption, and guidance information required.

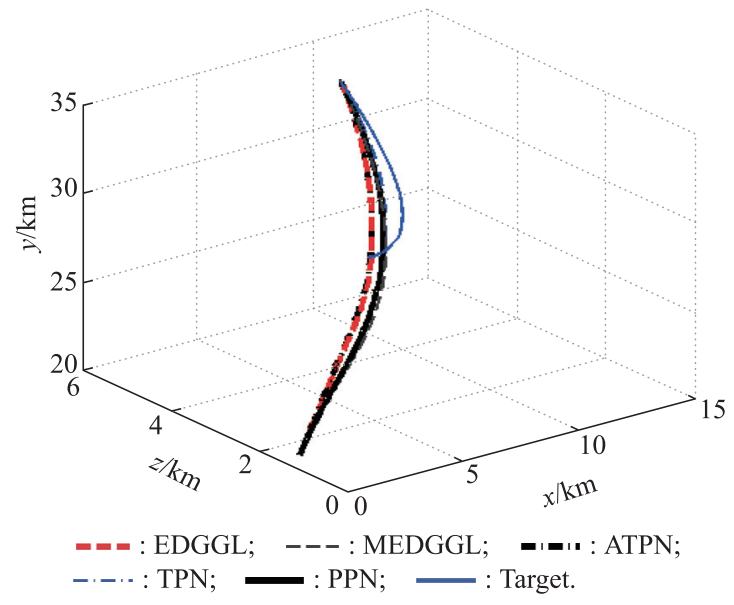

Fig. 4 Comparison of missile trajectories $\left(\mathbf{T}_{1}\right)$

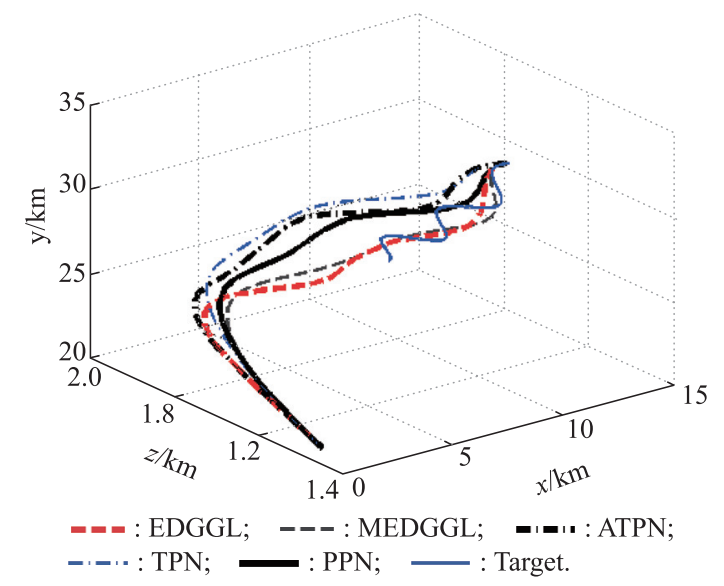

Fig. 5 Comparison of missile trajectories $\left(\mathbf{T}_{2}\right)$

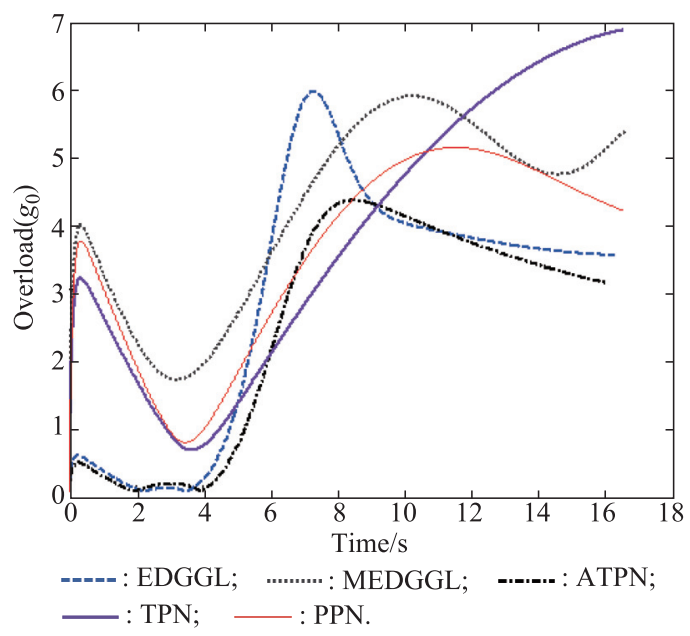

Fig. 6 Comparison of missile overloads $\left(\mathrm{T}_{1}\right)$

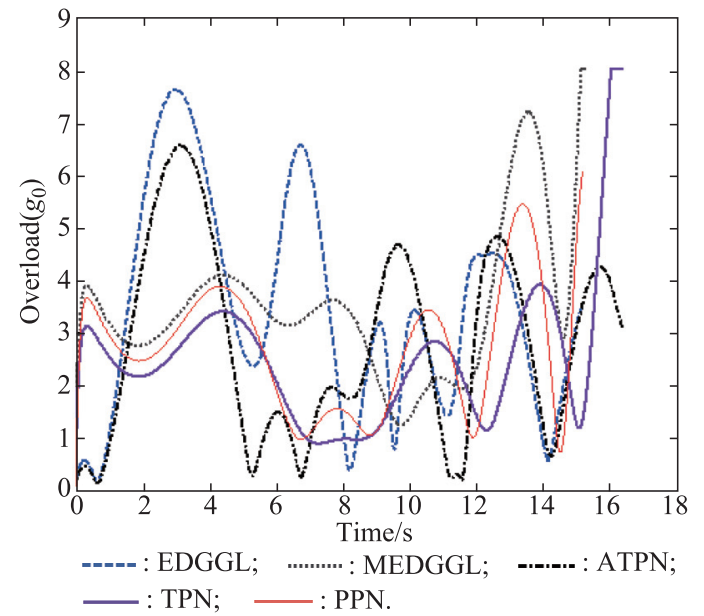

Fig. 7 Comparison of missile overloads $\left(\mathbf{T}_{2}\right)$

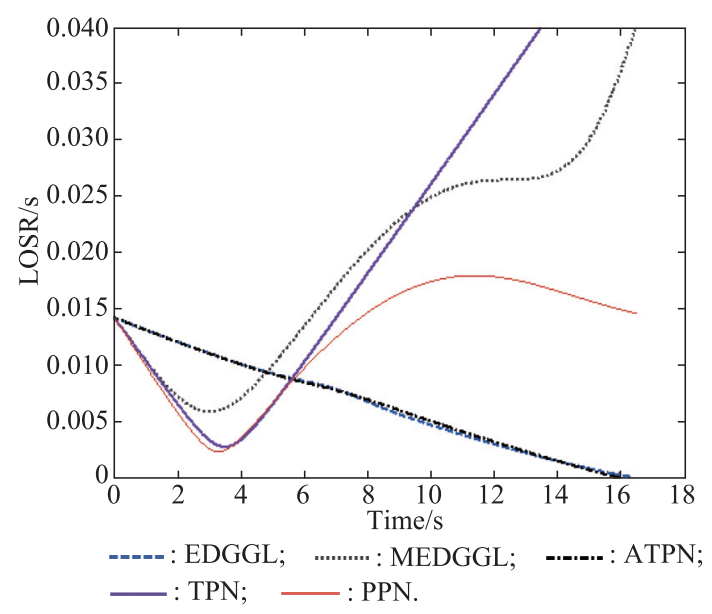

Fig. 8 Comparison of missile LOSRs ( $\left.\mathrm{T}_{1}\right)$

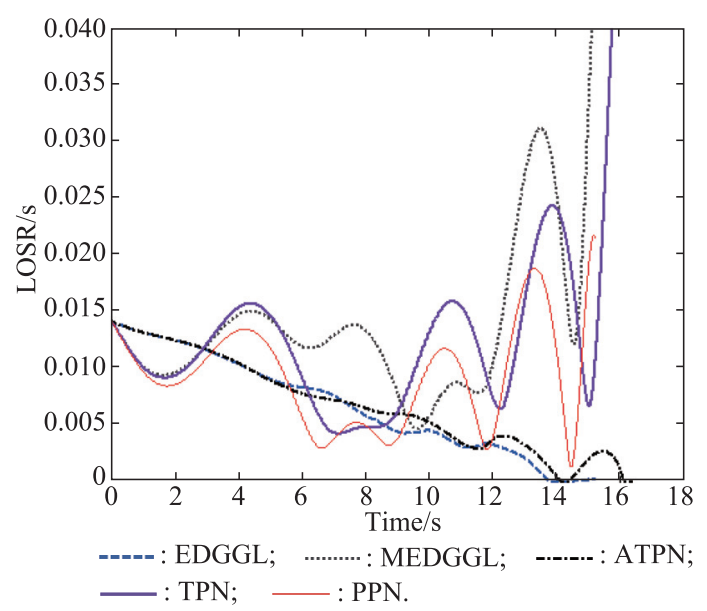

Fig. 9 Comparison of LOSRs $\left(\mathrm{T}_{2}\right)$

Scenario B The missile with a velocity disadvantage head-on intercepting target. The initial conditions of missile and target in $A x y z$ and two types of target maneuver are shown in Table 3. 
Table 3 Simulation settings in Scenario B

\begin{tabular}{ccc}
\hline Settings & Missile & Target \\
\hline Initial position/km & $(3.75,30,2.25)$ & $(33.75,45,2.25)$ \\
Initial speed/(km/s) & 2.1 & 2.7 \\
Initial pointing of $\boldsymbol{t}$ & $(0.4800,0.8703,0.1104)$ & $(-1,0,0)$ \\
Initial pointing of $\boldsymbol{n}$ & $(-0.5410,0.3927,-0.7437)$ & - \\
Maneuvering manner & - & $\mathrm{K}_{1}:\left(0, g_{0}, 2 g_{0}\right) ; \mathrm{K}_{2}:\left(0, g_{0}, 3 g_{0} \cos t\right)$ \\
\hline
\end{tabular}

From Table 3 , the initial $\boldsymbol{n}_{m} \cdot \boldsymbol{e}_{\theta}$ is 0.7999 , and the initial $r \omega$ is

$$
r_{0} \omega_{0}=0.1110<\left|\frac{v_{t 0}}{v_{m}}-1\right|=0.2857
$$

In (45) same as (44), $v_{t e}$ is replaced by $v_{t 0}$.

In the following simulations, the maximum overload of the missile is set as $12 g_{0}$, the seeker blind zone is defined as $500 \mathrm{~m}$, and the target successively employs the maneuvering manners of $\mathrm{K}_{1}$ and $\mathrm{K}_{2}$. The missile is guided by EDGGL and MEDGGL simultaneously compared with TPN, ATPN and PPN. The simulation results are shown in Figs. $10-15$ and Table 4.

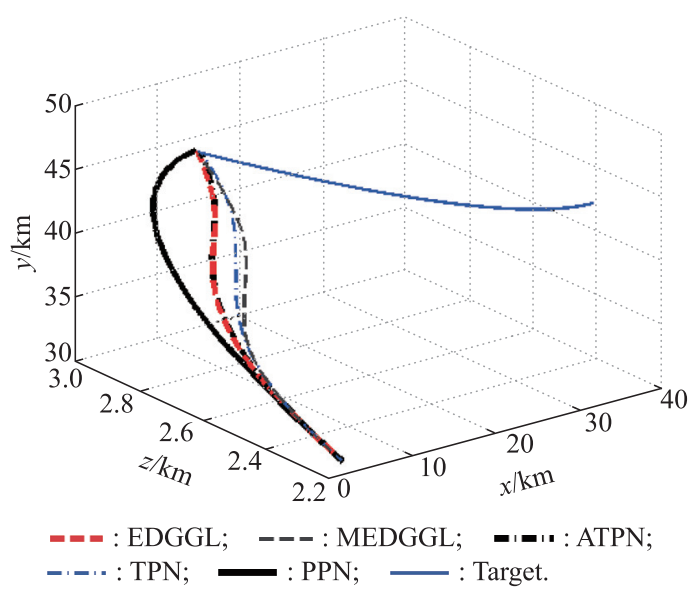

Fig. 10 Comparison of missile trajectories $\left(K_{1}\right)$

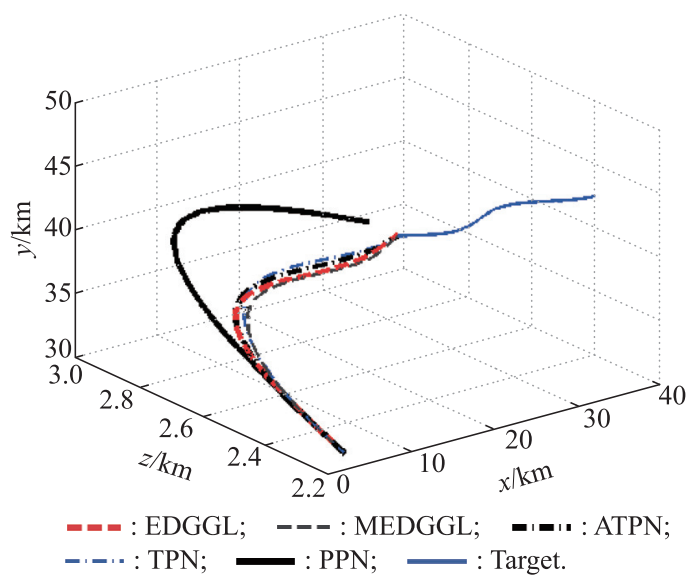

Fig. 11 Comparison of missile trajectories $\left(\mathrm{K}_{2}\right)$

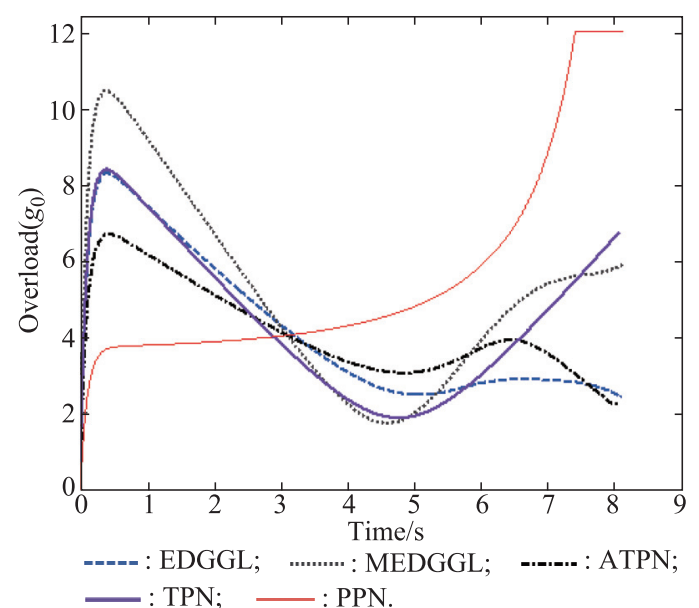

Fig. 12 Comparison of missile overloads $\left(K_{1}\right)$

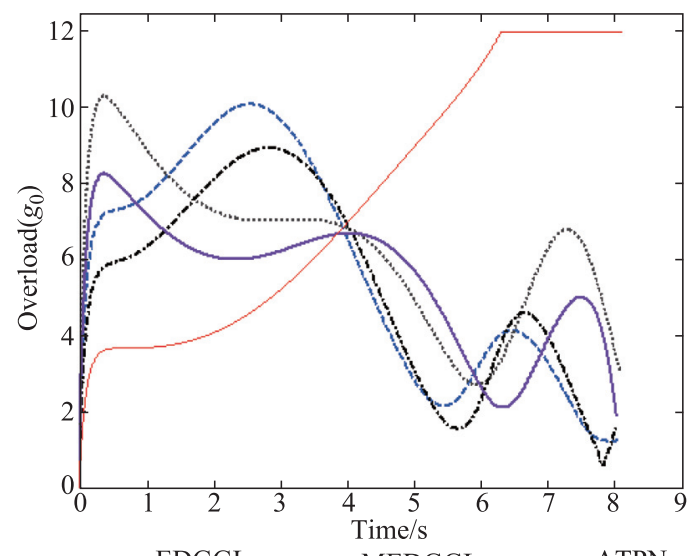

EDGGL

: MEDGGL;

-...... : ATPN;

: TPN $\quad$ : PPN

Fig. 13 Comparison of missile overloads $\left(K_{2}\right)$

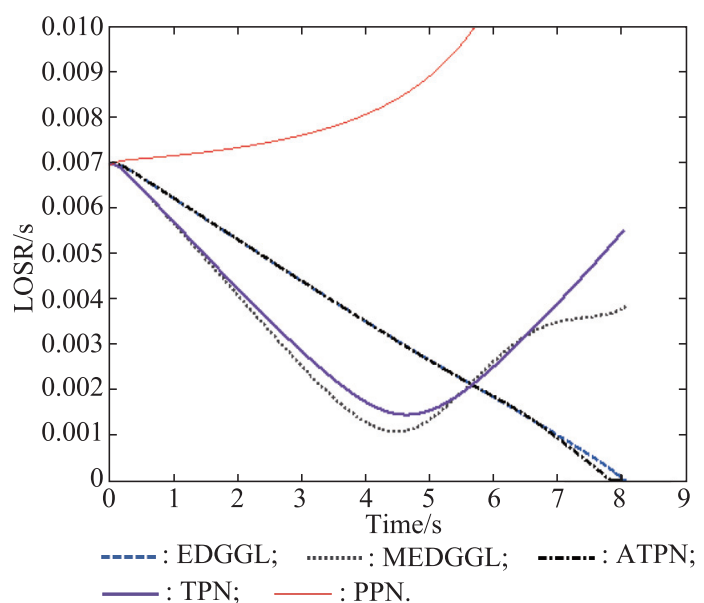

Fig. 14 Comparison of missile $\operatorname{LOSRs}\left(\mathrm{K}_{1}\right)$ 


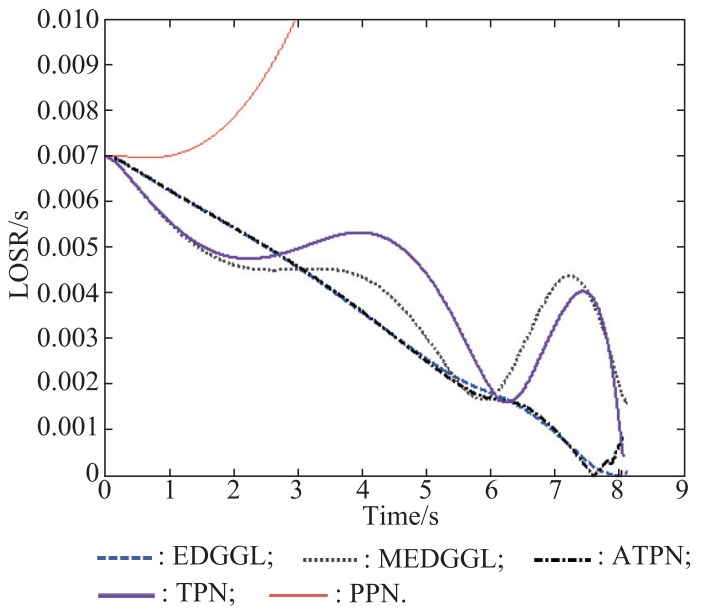

Fig. 15 Comparison of missile LOSRs $\left(K_{2}\right)$

Table 4 Comparisons of simulation results in Scenario $\mathrm{B}$

\begin{tabular}{cccccc}
\hline Guidance & \multicolumn{2}{c}{$\mathrm{K}_{1}$} & & \multicolumn{2}{c}{$\mathrm{K}_{2}$} \\
\cline { 2 - 3 } \cline { 5 - 6 } law & Miss distance $/ \mathrm{m}$ & $\Delta v /(\mathrm{m} / \mathrm{s})$ & & Miss distance $/ \mathrm{m}$ & $\Delta v /(\mathrm{m} / \mathrm{s})$ \\
\hline EDGGL & 0.1699 & 329.9139 & & 0.0945 & 456.0462 \\
MEDGGL & 0.3817 & 406.0167 & & 0.2528 & 503.4994 \\
ATPN & 0.2554 & 323.4191 & & 0.1385 & 418.0749 \\
TPN & 0.4972 & 350.2455 & & 0.1119 & 432.8559 \\
PPN & 27.0632 & 436.5607 & & 106.7757 & 594.2678 \\
\hline
\end{tabular}

Because of (31) and (37), in Table 4 the overload consumptions of EDGGL and MEDGGL are more than those of ATPN and TPN, respectively. Moreover, Table 4 reveals that the compensation of target maneuver has a little influence on lowering overload, which can be put down to the short intercept time of head-on high-speed flight and the relatively weak maneuver of high-speed targets. At the same time, the miss distances of MEDGGL and TPN can satisfy certain guidance precision. Thus, it is suggested that there is no need to introduce the acceleration compensation in engagement scenarios similar to Scenario B.
According to Fig. 14 and Fig. 15, the guidance laws except PPN are capable of suppressing LOSR, particularly EDGGL and ATPN. The engagement geometry of Scenario B exceeds the capture capability of PPN and the divergent LOSR, consequently, gives rise to the interception failure. If engagement scenarios like Scenario B occur outside the atmosphere, we can make a compromise to select TPN as the guidance law. However, as the target velocity inside the atmosphere becomes progressively faster, missiles gradually lose the advantage in velocity. Furthermore, targets may adopt certain maneuvering. In order to cope with this type of target, we have to employ the head-on intercept. On basis of the results in Scenario B, it is known that MEDGGL can be used to intercept the high-speed maneuvering target, instead of the ineffective PPN. Under the condition that the estimation of target acceleration is accurate, EDGGL can be chosen to lower overload consumption and miss distance.

In view of the simulation results in Scenarios $A$ and $B$, it is demonstrated that both EDGGL and MEDGGL are able to guide missile to pursue target with a velocity disadvantage and head-on intercept target with an advantage. Moreover, MEDGGL can deal with certain target maneuver. This dramatically extends the application range of DGGL.

\subsection{Demonstration of capture performance}

Numerical simulations are conducted to examine the reasonability of (41), while PPN is used as a benchmark guidance law. Based on the simulation settings in Scenario A, we give the different values of $r_{0} \omega_{0}$ by only adjusting the initial $\boldsymbol{t}_{m}$. According to (33), the initial $\boldsymbol{n}_{m}$ is computed. The rest of simulation settings are identical to Scenario A and the maneuvering manner of target is chosen as $T_{1}$. The four situations are listed in Table 5.

Table 5 Four situations for capture performance (Scenario A)

\begin{tabular}{cccc}
\hline Number & Initial $\boldsymbol{t}_{m}$ & Initial $\boldsymbol{n}_{m}$ & $r_{0} \omega_{0}$ \\
\hline A1 & $(0.6395,0.7543,0.1486)$ & $(0.4726,-0.2333,-0.8498)$ & $0.1491<0.6$ \\
A2 & $(0.5895,0.6543,0.4737)$ & $(0.6099,0.0240,-0.7921)$ & $0.4737<0.6$ \\
A3 & $(0.5005,0.6543,0.5669)$ & $(0.7067,0.0694,-0.7041)$ & $0.5743<0.6$ \\
A4 & $(0.4995,0.6043,0.6207)$ & $(0.6310,0.2372,-0.7387)$ & $0.6256>0.6$ \\
\hline
\end{tabular}

In Table 5, "0.6" is the value of $\left|v_{t 0} / v_{m}-1\right|$. Thus, apparently, A1, A2, and A3 meet the capture criterion, while A4 does not. The simulation results are presented in
Table 6. To illustrate the results, the change trends of closing velocity of EDGGL and MEDGGL are depicted in Fig. 16 and Fig. 17, respectively.

Table 6 Comparisons of simulation results (Scenario A)

\begin{tabular}{|c|c|c|c|c|c|c|}
\hline \multirow{2}{*}{ Number } & \multicolumn{2}{|c|}{ EDGGL } & \multicolumn{2}{|c|}{ MEDGGL } & \multicolumn{2}{|c|}{ PPN } \\
\hline & Miss distance $/ \mathrm{m}$ & $\Delta v /(\mathrm{m} / \mathrm{s})$ & Miss distance $/ \mathrm{m}$ & $\Delta v /(\mathrm{m} / \mathrm{s})$ & Miss distance/m & $\Delta v /(\mathrm{m} / \mathrm{s})$ \\
\hline A1 & 0.8937 & 453.6090 & 2.0458 & 678.6976 & 1.3584 & 571.5543 \\
\hline A2 & 0.7268 & 909.6130 & 2.1211 & 1057.0060 & 1.3228 & 817.5180 \\
\hline A3 & 0.5612 & 846.7647 & 3.3263 & 1133.8791 & 1.3420 & 914.9269 \\
\hline A4 & 111.8596 & 1231.0550 & 10.4188 & 1154.8961 & 1.3239 & 989.3020 \\
\hline
\end{tabular}




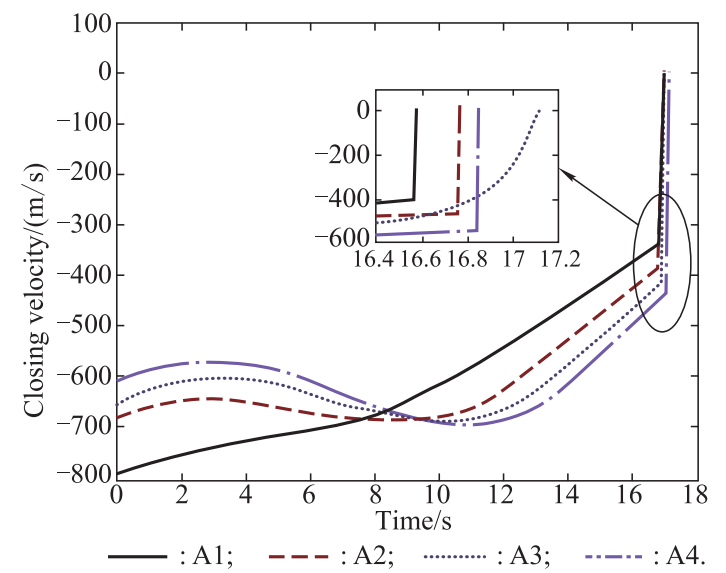

Fig. 16 Histories of EDGGL closing velocities $\left(\mathbf{T}_{1}\right)$

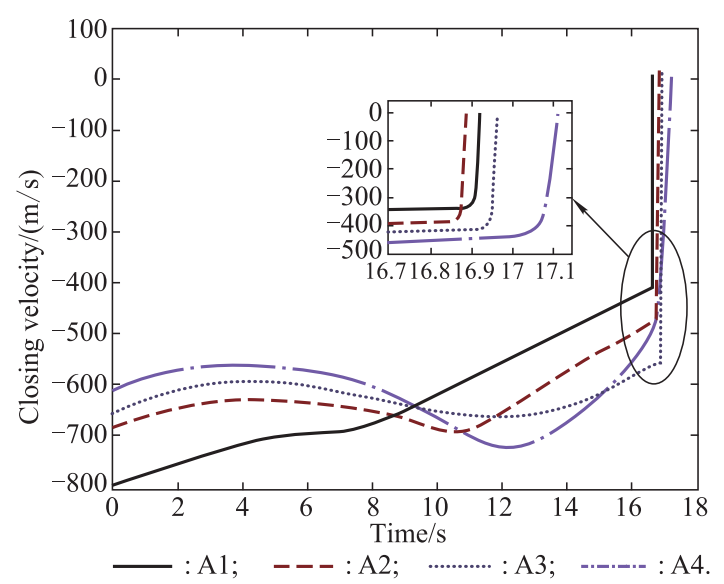

Fig. 17 Histories of MEDGGL closing velocities $\left(\mathrm{T}_{1}\right)$

From Table 6, it is known that the overload consump- tion $\Delta v$ generally becomes greater with the increase of $r_{0} \omega_{0}$ since $r_{0} \omega_{0}$ signifies the heading deviation from collision triangle and greater deviation is bound to require more overload to correct. What's more valuable is that when the capture criterion is not met, the miss distances of $\mathrm{A} 4$, for EDGGL and MEDGGL, abruptly increase, which represents the failure of interception. It can be observed from Fig. 16 and Fig. 17 that the trend of $\dot{r}$ gradually approaching to zero happens to A4 during the interception of drawing to a close. By contrast, the closing velocities of A1, A2, and A3 nearly jump to zero at the end of interception. This phenomenon in Fig. 16 is more distinct than that in Fig. 17 in that (41), for MEDGGL, is appropriate for the situation of $a_{t \theta}=0$. In accordance with the analysis in Section 5, the dissatisfaction of (41), in A4, leads to Case 1 being transformed into Case 2, not Case 4. In fact, (41) reveals the capability of correcting deviation of EDGGL and MEDGGL with the guarantee of capture. Additionally, it can be seen from Table 6 that PPN, with respect to the designed four situations, has the ability to accomplish the interceptions with relative small miss distances, which indicates the capture performance of PPN is better in terms of pursuing engagement.

Likewise, based on the simulation settings in Scenario $\mathrm{B}$, we provide the different values of $r_{0} \omega_{0}$ by only adjusting the initial pointing of $\boldsymbol{t}_{m}$. According to (33), the initial $\boldsymbol{n}_{m}$ is calculated. The reminder of simulation settings are same as Scenario $\mathrm{B}$ and the maneuvering manner of the target is chosen as $\mathrm{K}_{1}$. The four situations for capture performance are shown in Table 7.

Table 7 Four situations for capture performance (Scenario B)

\begin{tabular}{cccc}
\hline Number & Initial $\boldsymbol{t}_{m}$ & Initial $\boldsymbol{n}_{m}$ & $r_{0} \omega_{0}$ \\
\hline B1 & $(0.4800,0.8703,0.1104)$ & $(-0.5410,0.3927,-0.7437)$ & $0.1110<0.2857$ \\
B2 & $(0.4900,0.8480,0.2020)$ & $(-0.5388,0.4768,-0.6946)$ & $0.2051<0.2857$ \\
B3 & $(0.5130,0.8203,0.2529)$ & $(-0.5609,0.5433,-0.6247)$ & $0.2626<0.2857$ \\
B4 & $(0.5030,0.8103,0.3007)$ & $(-0.5232,0.5624,-0.6404)$ & $0.3100>0.2857$ \\
\hline
\end{tabular}

In Table 7, "0.285 7" is the value of $\left|v_{t 0} / v_{m}-1\right|$. Hence, obviously, B1, B2, and B3 satisfy the capture criterion, while B4 does not. The simulation results are listed in Ta- ble 8 . The change trends of closing velocity of EDGGL and MEDGGL are described in Fig. 18 and Fig. 19, respectively.

Table 8 Comparisons of simulation results (Scenario B)

\begin{tabular}{|c|c|c|c|c|c|c|}
\hline \multirow{2}{*}{ Number } & \multicolumn{2}{|c|}{ EDGGL } & \multicolumn{2}{|c|}{ MEDGGL } & \multicolumn{2}{|c|}{ PPN } \\
\hline & Miss distance/m & $\Delta v /(\mathrm{m} / \mathrm{s})$ & Miss distance/m & $\Delta v /(\mathrm{m} / \mathrm{s})$ & Miss distance $/ \mathrm{m}$ & $\Delta v /(\mathrm{m} / \mathrm{s})$ \\
\hline B1 & 0.1699 & 329.9139 & 0.3817 & 406.0167 & 27.0632 & 436.5607 \\
\hline B2 & 0.1605 & 804.1781 & 0.2769 & 804.9182 & 228.8570 & 766.1484 \\
\hline B3 & 947.6443 & 984.3798 & 703.4155 & 981.3417 & 615.8481 & 895.6205 \\
\hline B4 & 1922.6400 & 991.8535 & 1537.5724 & 990.4796 & 1089.6644 & 941.5258 \\
\hline
\end{tabular}




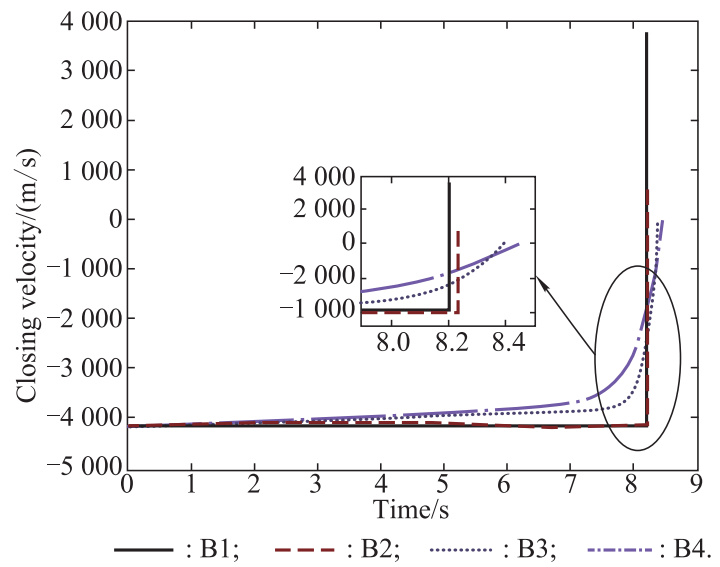

Fig. 18 Histories of EDGGL closing velocities $\left(K_{1}\right)$

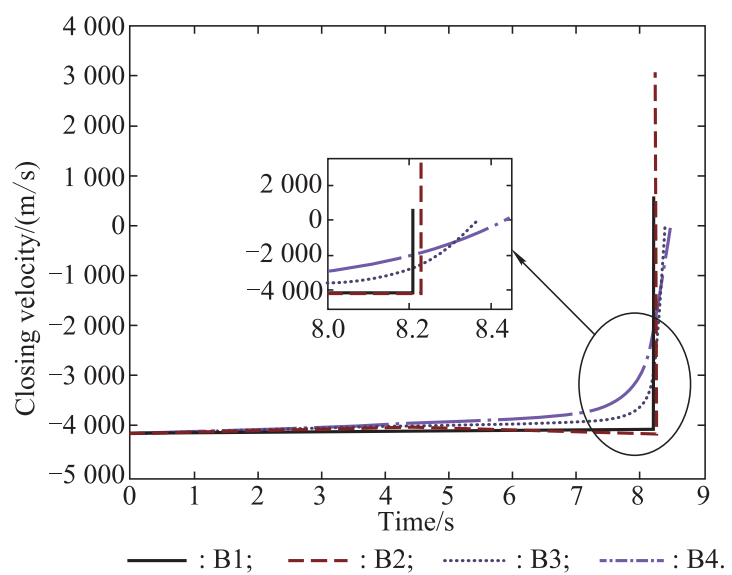

Fig. 19 Histories of MEDGGL closing velocities $\left(K_{1}\right)$

In Fig. 18 and Fig. 19, B3 and B4 encounter the misfortune that $\dot{r}$ is increasingly close to zero. Accordingly, the miss distances of B3 and B4 shoot up in Table 8. By contrast, the closing velocities of B1 and B2 directly jump to zero. Specially, the reason for the failure of B3, which meets the capture criterion, lies in the limitation of maximum overload. When $r_{0} \omega_{0}$ is closer to $\left|v_{t 0} / v_{m}-1\right|$, the required overload is saturated in longer duration. Therefore, (41) can be utilized to evaluate the handover accuracy from midcourse to terminal guidance and a lower value of $r_{0} \omega_{0}$ is better. In addition, PPN is invalid in terms of the devised four situations.

According to the above simulation results, we can conclude that the analysis about capture performance is correct and (41) is able to serve as a criterion for capture without regard to other interception factors.

\section{Conclusions}

This paper is devoted to abandoning the unnecessary assumptions made on missile and target in the derivation of DGGL and extend the application range of DGGL. By means of introducing the LOS rotation coordinate system, the process of derivation is simplified and its physical significances are more explicit. As a result, the EDGGL is obtained. Referring to TPN, we further from EDGGL derive MEDGGL without target maneuvering information included and the determination method is proposed of the applied direction of commanded missile acceleration. Then, the conservative criterion for capture is given and, irrespective of other interception factors, can be used to judge capture or miss. By constructing the three-dimensional pursuing and head-on intercepting scenarios, the effectiveness of EDGGL and MEDGGL is validated and the application range of DGGL is greatly extended. Simultaneously, it is found that EDGGL and MEDGGL can be used to intercept high-speed maneuvering targets that PPN cannot deal with inside the atmosphere. Finally, future work will concentrate on the following aspects: i) making a detailed comparison and analysis of the robustness of keeping $\boldsymbol{n}_{m} \cdot \boldsymbol{e}_{\theta}$ constant in EDGGL and MEDGGL; ii) combining (27) with (36) to constitute a new DGGL and then selecting an observer to estimate $a_{t \theta}$; iii) how to utilize current control systems to realize EDGGL and MEDGGL.

\section{References}

[1] SHNEYDOR N A. Missile guidance and pursuit: kinematics, dynamics and control. Chichester: Horwood Publishing, 1998.

[2] SHUKLA U S, MAHAPATRA P R. The proportional navigation dilemma-pure or true. IEEE Trans. on Aerospace and Electronic Systems, 1990, 26(2): 382-392.

[3] YUAN P J, CHERN J S. Ideal proportional navigation. Journal of Guidance, Control, and Dynamics, 1992, 15(5): $1161-$ 1165 .

[4] YANG C D, YANG C C. A unified approach to proportional navigation. IEEE Trans. on Aerospace and Electronic Systems, 1997, 33(2): $557-567$.

[5] ZARCHAN P. Tactical and strategic missile guidance. Reston, Virginia: American Institute of Aeronautics and Astronautics, 2012: $163-171$.

[6] CHEN L, ZHANG B. Novel TPN control algorithm for exoatmospheric intercept. Journal of Systems Engineering and Electronics, 2009, 20(6): 1290-1295.

[7] LI K B, ZHANG T T, CHEN L. Ideal proportional navigation for exoatmospheric interception. Chinese Journal of Aeronautics, 2013, 26(4): 976-985.

[8] CHO N, KIM Y. Modified pure proportional navigation guidance law for impact time control. Journal of Guidance, Control, and Dynamics, 2016, 39(4): 852-872.

[9] STRUIK D J. Lectures on classical differential geometry. New York: Dover Publications, 1988.

[10] CHO N, KIM Y, PARK S. Three-dimensional nonlinear differential geometric path-following guidance law. Journal of Guidance, Control, and Dynamics, 2015, 38(12): 2366-2385.

[11] MENG Y H, CHEN Q F, NI Q. A new geometric guidance approach to spacecraft near-distance rendezvous problem. Acta Astronautica, 2016, 129: 374-383.

[12] CHIOU Y C, KUO C Y. Geometric approach to threedimensional missile guidance problem. Journal of Guidance, Control, and Dynamics, 1998, 21(2): 335 -341.

[13] KUO C Y, CHIOU Y C. Geometric analysis of missile guid- 
ance command. IEE Proceedings-Control Theory and Applications, 2000, 147(2): $205-211$.

[14] KUO C Y, SOETANTO D, CHIOU Y C. Geometric analysis of flight control command for tactical missile guidance. IEEE Trans. on Control Systems Technology, 2001, 9(2): 234-243.

[15] LI C Y, JING W X. New results on three-dimensional differential geometric guidance and control problem. Proc. of the AIAA Guidance, Navigation, and Control Conference and Exhibit, 2006: AIAA 2006-6086.

[16] LI C Y, JING W X, WANG H, et al. Iterative solution to differential geometric guidance problem. Aircraft Engineering and Aerospace Technology, 2006, 78(5): 415 - 425.

[17] LI C Y, JING W X. Application of PID controller to 2D differential geometric guidance problem. Journal of Control Theory and Applications, 2007, 5(3): 285-290.

[18] LI C Y, JING W X. Fuzzy PID controller for 2D differential geometric guidance and control problem. IET Control Theory and Applications, 2007, 1(3): $564-571$.

[19] LI C Y, JING W X, WANG H, et al. Development of flight control system for 2D differential geometric guidance and control problem. Aircraft Engineering and Aerospace Technology, 2007, 79(1): 60-68.

[20] LI C Y, JING W X, WANG H, et al. A novel approach to the 2D differential geometric guidance problem. Transactions of the Japan Society for Aeronautical and Space Sciences, 2007, 50(167): $34-40$.

[21] LI C Y, JING W X, WANG H, et al. Gain-varying guidance algorithm using differential geometric guidance command. IEEE Trans. on Aerospace and Electronic Systems, 2010, 46(2): 725 - 736

[22] LI K B, CHEN L, BAI X Z. Differential geometric modeling of guidance problem for interceptors. Science China Technological Sciences, 2011, 54(9): 2283-2295.

[23] LI K B, CHEN L, TANG G J. Improved differential geometric guidance commands for endoatmospheric interception of highspeed targets. Science China Technological Sciences, 2013, 56(2): $518-528$.

[24] LI K B, CHEN L, TANG G J. Algebraic solution of differential geometric guidance command and time delay control. Science China Technological Sciences, 2015, 58(3): 565-573.

[25] YE J K, LEI H M, XUE D F, et al. Nonlinear differential geometric guidance for maneuvering target. Journal of Systems Engineering and Electronics, 2012, 23(5): 752-760.

[26] MA Y W, ZHANG W H, LONG H. Intelligent guidance method based on differential geometric guidance command and fuzzy self-adaptive guidance law. Journal of Intelligent and Fuzzy Systems, 2015, 29(6): 2527-2536.

[27] MA Y W, ZHANG W H. Differential geometric guidance command with finite time convergence using extended state observer. Journal of Central South University, 2016, 23(4): $859-868$.

[28] ARIFF O, ZBIKOWSKI R, TSOURDOS A, et al. Differential geometric guidance based on the involute of the target's trajectory. Journal of Guidance, Control, and Dynamics, 2005, 28(5): $990-996$.

[29] WHITE B A, ZBIKOWSKI R, TSOURDOS A. Direct intercept guidance using differential geometric concepts. IEEE Trans. on Aerospace and Electronic Systems, 2007, 43(3): 899-919.

\section{Biographies}

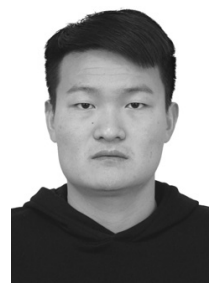

HUANG Jingshuai was born in 1993. He received his B.S. degree in aircraft design and engineering from Nanjing University of Aeronautics and Astronautics in 2013 and M.S. degree in aeronautical and astronautical science and technology form $\mathrm{Na}-$ tional University of Defense Technology in 2015, respectively. Now, he is a Ph.D. candidate in National University of Defense Technology. His research interests are in flight vehicle dynamics, intercept guidance and control.

E-mail: hjs_nudt@126.com

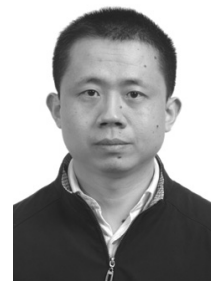

ZHANG Hongbo was born in 1981. He received his $\mathrm{Ph} . \mathrm{D}$. degree in aeronautics and astronautics from National University of Defense Technology in 2009. Currently, he is an associate professor and a master tutor in the College of Aerospace Science and Engineering, National University of Defense Technology. His research interests include orbit dynamics and control, flight vehicle dynamics and control, and entry guidance.

E-mail: zhanghb1304@ nudt.edu.cn

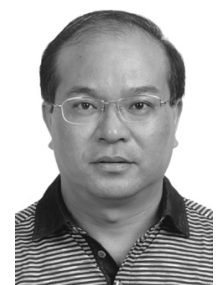

TANG Guojian was born in 1964. He received his $\mathrm{Ph} . \mathrm{D}$. degree in control theory and engineering from National University of Defense Technology in 2000. At present, he is a professor and doctoral supervisor in the College of Aerospace Science and Engineering, National University of Defense Technology. His research interests are mainly in flight vehicle dynamics, guidance, and control.

E-mail: tangguojian@nudt.edu.cn

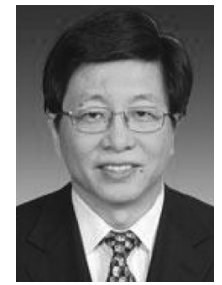

BAO Weimin was born in 1960. Currently, he is an academician of Chinese Academy of Sciences and a researcher in China Aerospace Science and Technology Corporation. In addition, he is a doctoral supervisor in the College of Aerospace Science and Engineering, National University of Defense Technology. His research interests are mainly in flight vehicle overall design.

E-mail: baoweimin@cashq.ac.cn 